\title{
Chemokines and HIV replication
}

SIR - Three chemotactic peptides belonging to the $\beta$-chemokine group of cytokines, RANTES, MIP- $1 \alpha$, and MIP$1 \beta$, inhibit the infection of $\mathrm{CD} 4^{+} \mathrm{T}$ cells by primary HIV-1 strains ${ }^{1}$. Although all three peptides suppress HIV-1 replication in $\mathrm{T}$ cells, they do so with different efficiency, with RANTES being the most active, followed by MIP-1 $\beta$, then MIP- $1 \alpha^{1}$. Here we demonstrate that, in sharp contrast to their observed antiviral effects in $\mathrm{T}$ cells, $\beta$-chemokines actually stimulate the replication of primary HIV-1 strains in macrophages, another major target of this virus. The magnitude of stimulation is dependent on the cell donor and HIV-1 strain used, and varies for different $\beta$ chemokine peptides.

In addition to $\mathrm{T}$ lymphocytes, monocyte/macrophages are a rich source of $\beta$ chemokines in the body ${ }^{2}$. HIV-1 infection itself upregulates $\beta$-chemokine expression in monocytes both in vitro and in vivo ${ }^{3}$. In the light of their anti-HIV effects on $\mathrm{T}$ cells, the release of these peptides by macrophages in response to HIV-1 infection might reflect a defensive manoeuvre of the immune system. To test this hypothesis, we performed experiments to assess the anti-HIV-1 activity of $\beta$-chemokine peptides in monocyte cultures, and the effect of chemokine peptides on HIV-1 replication in $\mathrm{T}$ lymphocytes.

We used two primary HIV-1 isolates that replicate both in $\mathrm{T}$ lymphocytes and monocytes, 92US657 and 92US660 (obtained from NIH AIDS Research and Reference Reagent Program), for infections. We isolated lymphocytes from the same donors as monocytes by passing non-adherent cells through a T-cell enrichment column ( $R \& D$, Minneapolis) resulting in a $95 \%$ pure $\mathrm{CD}^{+}+\mathrm{CD}^{+} \mathrm{T}$ cell population. As expected, RANTES,
MIP-1 $\beta$ and MIP-1 $\alpha$ (all peptides from PeproTech Inc.) suppress replication of both isolates in $\mathrm{T}$ lymphocytes, with a $50 \%$ inhibitory concentration $\left(\mathrm{IC}_{50}\right)$ of 5 , 15 and $35 \mathrm{ng} \mathrm{ml}^{-1}$, respectively, in good agreement with previously published results ${ }^{1}$. At $500 \mathrm{ng} \mathrm{ml}^{-1}$, each chemokine inhibited HIV-1 replication by more than $95 \%$, and this concentration was chosen for testing chemokines' effects on HIV-1 infection of macrophages.

In contrast, we observed an enhancing, rather than an inhibitory, activity of all three $\beta$-chemokines on the replication of both HIV-1 strains in macrophage cultures prepared from two donors (see figure). We observed this enhancing effect of $\beta$-chemokine peptides throughout the course of infection; data in the figure show results obtained on day 15 postinfection when virus replication reached the peak. This effect is dose-dependent (data not shown) and is not the result of contaminating lipopolysaccharide in chemokine preparations (less than $0.1 \mathrm{ng}$ per $\mu g$ of peptide) as similar amounts of lipopolysaccharide either have no effect or suppress HIV-1 replication in macrophage cultures (not shown). We obtained similar results (not shown) with another HIV-1 strain, HIV-1 $1_{\mathrm{ADA}}$, and $\beta$ chemokine peptides from a different source $(R \& D)$, indicating that the observed phenomenon does not result from peculiarities of the chemokine preparations but rather reflects a general feature of these molecules.

Dragic et al. ${ }^{4}$ have also observed failure of $\beta$-chemokines to inhibit HIV-1 infection of primary monocytes. However, these authors did not detect any stimulatory effect of these peptides on viral replication. The different results could be due to the source of virus used for infection:

Effect of $\beta$-chemokine peptides on HIV-1 replication in monocyte cultures. Dark boxes, controls; light hatching MIP-1 $\alpha$; heavy hatching, MIP-1 $\beta$; reverse hatching, RANTES. We cultured macrophage cultures prepared from PBMCs of two donors (a and $b$ ) by adherence to plastic ${ }^{3}$ for 7 days and then infected them with HIV-1 $\left(2 \times 10^{4}\right.$ c.p.m. of

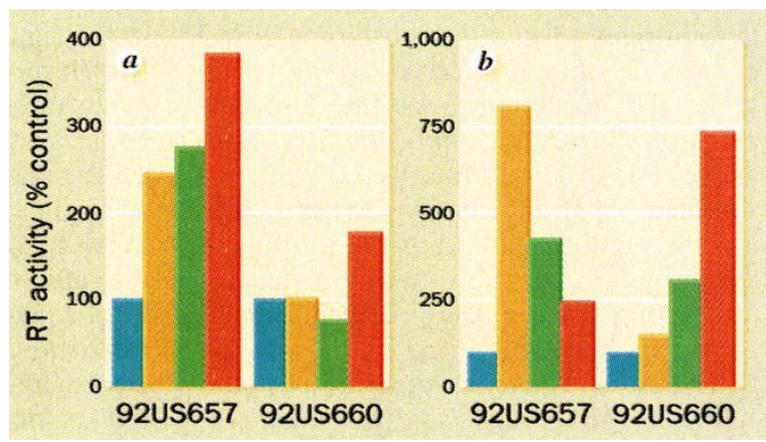

reverse transcriptase

(RT) per $10^{6}$ cells in $1 \mathrm{ml}$ medium) in the presence of $500 \mathrm{ng} \mathrm{ml}^{-1}$ chemokine $\beta$-peptides. We replaced the culture medium every 3 days with fresh medium containing $500 \mathrm{ng} \mathrm{ml}^{-1}$ chemokines. Fifteen days after infection, we tested culture supernatants for reverse transcriptase activity. Results are presented as per cent of RT activity in untreated (control) culture supernatants taken as $100 \%$. RT activity for the controls is $5.8 \times 10^{5}$ c.p.m. ml ${ }^{-1}$ for 92US657 and $2.8 \times 10^{5}$ for 92US660. Blue, control; orange, MIP-1 $\alpha$; green, MIP-1 $\beta$; red, RANTES.
Dragic et al. used recombinant HIV-1 strains, whereas our results were obtained with primary isolates. As shown in the figure, strain differences significantly affect the magnitude of the stimulation. Although the observed stimulatory effect of $\beta$-chemokines on HIV-1 replication could be a by-product of their ability to activate macrophages, other mechanisms must be also involved, as MIP- $1 \alpha$ is a much more potent stimulatory agent than MIP- $1 \beta^{5}$, yet their effect on HIV-1 replication is comparable in most cases ( $a$ in the figure).

Taken together, these findings suggest that the ability of $\beta$-chemokine peptides to either inhibit or stimulate HIV-1 replication is cell-type dependent. Failure of chemokines to inhibit HIV-1 replication in macrophages despite the presence on these cells of CCR-5, a $\beta$-chemokine receptor and a co-receptor for $\mathrm{HIV}-1^{4,6}$, indicates that a different co-receptor which does not bind $\beta$-chemokines might be involved in HIV-1 infection of macrophages. Because HIV-1 infection induces $\beta$-chemokine expression in monocytes $^{3}$, the infected immune system might actually benefit by allowing tempered viral replication in these cells (which are less susceptible to the cytopathic effects of infection than T cells ${ }^{7}$ ), so that sufficient amounts of the $\beta$ chemokines can be produced to inhibit vigorous virus replication in T-lymphocyte populations. On the other hand, high levels of $\beta$-chemokine peptides could produce harmful results by enhancing HIV-1 replication in macrophages and/or intensifying virusinduced inflammation, as demonstrated for Coxsackie and influenza viruses ${ }^{8}$. These considerations should be taken into account when considering the use of $\beta$-chemokines as anti-HIV therapeutic agents.

\section{Helena Schmidtmayerova*,} Barbara Sherry \& Michael Bukrinsky The Picower Institute for Medical

Research,

Manhasset, New York 11030, USA

e-mail: mbukrinsky@picower.edu

* Also at: Institute of Virology, Slovak Academy of Sciences. 84246 Bratislava, Slovakia.

1. Cocchi, F. et al. Science 270, 1811-1816 (1995).

2. Sherry, B. \& Cerami, A. Curr. Opin. Immunol. 3, 56-60 (1991).

3. Schmidtmayerova, H. et al. Proc. Natl Acad. Sci. USA 93, 700-704 (1996).

4. Dragic, T. et al. Nature 381, 667-673 (1996).

5. Fahey, T. J. et al J. Immunol 148, 2764-2769 (1992).

6. Deng, $H$. et al. Nature 381, 661-666 (1996).

7. Gendelman, H. E. et al. AIDS 3, 475-495 (1989).

8. Cook, D. N. et al. Science 269, 1583-1585 (1995).

\section{Scientific Correspondence}

Scientific Correspondence is intended to provide a forum in which readers may raise points of a scientific character. Priority will be given to letters of fewer than 500 words and five references. 